\title{
Tidal breakup of the nucleus of Comet Shoemaker-Levy 9
}

\author{
By ZDENEK SEKA NINA
}

Jet Propulsion Laboratory, California Institute of Technology, Pasadena, CA 91109, USA

The breakup of Comet Shoemaker-Levy 9 is discussed both in the context of splitting as a cometary phenomenon, comparing this object with other split comets, and as an event with its own idiosyncrasies. The physical appearance of the comet is described, features diagnostic of the nature of tidal splitting are identified, and the implications for modelling the event are spelled out. Among the emphasized issues is the problem of secondary fragmentation, which documents the comet's continuing disintegration during 1992-94 and implies that in July 1992 the parent object split tidally near Jupiter into 10-12, not 21, major fragments. Also addressed are the controversies involving models of a strengthless agglomerate versus a discrete cohesive mass and estimates for the sizes of the progenitor and its fragments.

\section{Introduction}

Splitting is a relatively common phenomenon among comets, even though its detection is observationally difficult because companions are almost invariably very diffuse objects with considerable short-term brightness variations. Comet Shoemaker-Levy 9's behavior was generally less erratic than that of an average split comet, which may have in part been due to a major role of large-sized dust. The breakup products that contributed most significantly to the comet's total brightness are referred to below as components, or, because of their diffuse appearance, as condensations, both common terms of cometary phenomenology. The terms nuclei and fragments are instead reserved for genuine solid bodies of substantial dimensions ( $\gtrsim 1 \mathrm{~km}$ across) that were "hidden" in the condensations. A condensation may contain many fragments or nuclei, besides large amounts of material of subkilometer-sized and smaller particulates, the entire population of which is characterized by a certain size distribution function.

A total of 21 split comets had been documented in the literature by 1980 (for a review, see Sekanina 1982) and eleven additional ones have been reported since. Of these recent entries, fully eight are or were short-period comets (79P/du Toit-Hartley, 108P/Ciffréo, 101P/Chernykh, D/Shoemaker-Levy 9, P/Machholz 2, 51P/Harrington, 73P/Schwassmann-Wachmann 3, and the parent of $42 \mathrm{P} /$ Neujmin 3 and $53 \mathrm{P} /$ Van Biesbroeck); two are (or were) "old" comets (of which Takamizawa-Levy 1994 XIII = C/1994 G1 is one, while the breakup products of the other were discovered as two separate objects, Levy $1987 \mathrm{XXX}=\mathrm{C} / 1988 \mathrm{~F} 1$ and Shoemaker-Holt $1988 \mathrm{III}=\mathrm{C} / 1988 \mathrm{~J} 1$, orbiting the Sun in virtually identical paths with a period of $\sim 14,000$ years and passing through perihelion $2 \frac{1}{2}$ months apart); and one is a "new" comet from the Oort cloud (Wilson 1987 VII $=\mathrm{C} / 1986 \mathrm{P} 1$ ). Shoemaker-Levy 9 was unique among all the split comets in that the maximum number of condensations observed at the same time was by far the largest. It is shown below, however, that without the italicized qualification the statement would not be valid.

Comet Shoemaker-Levy 9 is one among only a few multiple comets that are known to have fragmented due primarily -if not entirely - to the action of tidal forces during their extremely close encounters with Jupiter or the Sun. Besides Shoemaker-Levy 9, direct evidence exists for $16 \mathrm{P} /$ Brooks 2 , which missed Jupiter by one planet's radius above the cloud tops in 1886; and for two or three members of the sungrazing comet 
group (1882 II = C/1882 R1, Ikeya-Seki 1965 VIII = C/1965 S1, possibly also Pereyra $1963 \mathrm{~V}=\mathrm{C} / 1963 \mathrm{R} 1$ ), whose perihelia were located within $\frac{2}{3}$ the Sun's radius above the photosphere. For other split comets, the nature of the disruption mechanism is not fully understood, although jettisoning of pancake-shaped fragments of an insulating mantle from the nuclear surface by stresses, built up unevenly beneath it, is consistent with the evidence suggesting that these comets "peel off" rather than break up (Sekanina 1982).

\section{The number of condensations}

The detected number of Shoemaker-Levy 9's condensations depended not only on the imaging circumstances and the instrument used, but also on the time of observation, because some of the condensations disappeared with time while others began to develop companions of their own. Accounts of high-resolution observations indicate that no more than 22 condensations were detected at a time. On the other hand, the combined number of condensations reported on visually inspected images obtained at various times appears, collectively, to total 25 . This is short of the record held by the progenitor of the group of sungrazing comets, from which all the observed members were found by Marsden (1989) to derive. The known sungrazers represent at least three generations of fragmentation products and include: (i) four members discovered between 1843 and 1887, one of which (1882 II) was observed after perihelion to have at least five components and another (1887 I = C/1887 B1) always appeared as a headless object (Kreutz 1888); (ii) four members discovered between 1945 and 1970; (iii) six members detected with a coronagraph onboard the SOLWIND spacecraft between 1979 and 1984; (iv) 10 members detected with a coronagraph onboard the Solar Maximum Mission spacecraft between 1987 and 1989; and (v) any possible precursor objects, of which an uncertain orbit exists for one (the comet of $1668=\mathrm{C} / 1668 \mathrm{E} 1$ ) and very little information on two more (the comets of 1106 and $371 \mathrm{BC}$ ). Excluding the dubious companion to $1963 \mathrm{~V}$, a few unlikely candidates in the 17th through 19th centuries (for an overview, see Marsden 1967), and the comets of 1106 and $371 \mathrm{BC}$, one still finds a total of 29 comets and companions-a number that moderately exceeds the 25 condensations of Shoemaker-Levy 9 -observed over a period of nearly 150 years. However, if this system of comet parentage is accepted, the number of catalogued split comets should be decreased by one, because 1882 II and 1965 VIII would not then be listed as separate entries. On the other hand, if only first-generation products of an object should be counted, the number of sungrazers would drop, but so would the number of condensations of comet Shoemaker-Levy 9, as discussed in some detail in Sec. 4.3.

\section{Appearance of comet Shoemaker-Levy 9}

The comet's condensations were all aligned in an essentially rectilinear configuration, which extended almost perfectly along a great circle of the projected orbit and whose appearance has often been fittingly compared to a string of pearls. In the technical literature, the collection of the condensations is usually referred to as the nuclear train or just the train. Even though the condensations were the most prominent features contributing substantially to the total brightness, significant amounts of material were also situated in between them, along the train's entire length.

The comet further exhibited three other kinds of morphological features. Extending from the train on either side were trails or wings, of which the east-northeastern one appeared to be slightly inclined relative to the train. Subtending a relatively small angle with the train and pointing generally to the west was a set of straight, narrow tails, 
whose roots coincided with the distinct condensations in the train. These parallel tails were immersed in, and on low-resolution images gradually blended with, an enormous, completely structureless sector of material, which was stretching to the north of its sharp boundary delineated by the nuclear train and the two trails.

\subsection{The nuclear train}

To describe the train's structure in detail, two notations were proposed to identify the condensations. The system introduced by Sekanina et al. (1994; hereafter referred to as Paper 1) has been employed, especially after the impacts, almost universally: the easternmost condensation, the first to crash, was named $\mathrm{A}$; the westernmost, which crashed the last, $\mathrm{W}$. The letters I and $\mathrm{O}$ were excluded to avoid any confusion with the symbols used for the respective digits. The relationship between this notation and Jewitt et al.'s (1993) system, which numbers the condensations, is: $\mathrm{A} \equiv 21, \mathrm{~B} \equiv 20, \ldots, \mathrm{W} \equiv 1$.

The train's length, defined by the projected distance between the condensations $A$ and $\mathrm{W}$, continuously grew with time, from $\sim 50$ arcsec shortly after discovery in late March to almost 70 arcsec by mid-July of 1993, to more than 2 arcmin by the beginning of 1994, to about 5.5 arcmin by early May, and to some 10 arcmin, equivalent to a projected distance of more than 2 million $\mathrm{km}$, by early July 1994. The train's enormous extent was reflected in the time span of 5.5 days between the first and the last impacts: July 16.84 UT for A and July 22.34 UT for W (Chodas \& Yeomans 1994).

The train's orientation varied relatively insignificantly during the period of more than one year between discovery and collision. The position angle, measured in the direction from A to W, was within $1^{\circ}$ of $256^{\circ}$ between late March and mid-July 1993, decreasing to $245^{\circ}$ by the beginning of 1994 , reaching a minimum of $241^{\circ}$ in early March, a maximum of $244^{\circ}$ at the beginning of June, and decreasing again, at an accelerating rate, to $\sim 240^{\circ}$ by mid-July 1994 .

A detailed analysis of the alignment of the individual condensations showed that five of them-B, J, M, P (later resolved into P1 and P2; see Sec. 3.2), and T-exhibited small but detectable off-train deviations on high-resolution images as early as MarchJuly 1993, during the first four months after discovery (Table 8 of Paper 1). From a comparison of the 1993 pre- and post-conjunction observations, it became apparent that this group of "anomalous" condensations also included F. And more recently, with the use of a large number of 1994 observations, this category of condensations grew further, now also encompassing G2, N, Q2 (see Sec. 3.2), U, and V. Thus, eleven (or more) of the condensations were found to deviate noticeably from the nearly perfectly aligned on-train condensations. The status of some of them, in particular C and/or D, is not entirely clear to this time.

\subsection{Physical evolution of the train}

Two of the condensations, J and M, had only been detected by Jewitt et. al. (1993) on four occasions between late March and mid-July 1993. They are not apparent on the images taken with the Hubble Space Telescope (HST) on July 1, 1993 (Weaver et al. 1994) and were not reported at any time during 1994. An unpublished account of a possible detection of J on the comet's image taken at Mauna Kea on December 14, 1993 does not appear to be correct.

The first signs of impending dramatic changes in the appearance of the train became evident on the July 1993 images obtained with the HST (Weaver et al. 1994). While the components $\mathrm{J}$ and $\mathrm{M}$ vanished, the condensation $\mathrm{Q}$, the brightest at the time, appeared to have a faint, diffuse companion some $0.3 \mathrm{arcsec}$ away at a position angle of $\sim 30^{\circ}$. At the location of the condensation $\mathrm{P}$ two very diffuse nebulosities can be seen, less than 
1 arcsec apart and aligned approximately with the train. The condensation L may also have exhibited a companion nebulosity to the north. The spatial brightness distribution in the condensations was reported by Weaver et al. to be significantly flatter than the inverse first power of distance from the center, with no molecular emissions in the spectral region between 2220 and $3280 \AA$.

By late January 1994, the time of the next HST observation (Weaver et al. 1995), the $P-Q$ region had developed considerably. The condensation $Q$ was manifestly broken into two, a brighter Q1 and a fainter Q2, 1.2 arcsec apart and the fainter to the northnorthwest of the brighter. The condensation $\mathrm{P}$ also consisted of two widely separated nebulosities. The fainter and poorly condensed component, $\mathrm{P} 1$, was 4.2 arcsec from Q1 and almost exactly to the north, while the brighter, P2, was 5.0 arcsec from Q1 in the north-northeastern direction. The separation of P1 from P2 was 2.2 arcsec, the former nearly to the west of the latter. Both components looked elongated: P1 very distinctly to the west-northwest, in the direction of the tail; P2 less noticeably to the southwest. Another significant development was the issuance, in the southward direction, of a bright "spur" from the condensation S (Weaver 1994). The spatial distribution in the condensations became steeper than the inverse first power of distance. The appearance of the nuclear train, except for the condensation W, is shown in Fig. 1.

Further morphological changes were noticed on the HST images of the comet taken on March 29-30, 1994. The condensation P2 had become double, the fainter component appearing rather diffuse, and the spur of $S$ had grown fainter (Weaver 1994; Weaver et al. 1995). While most condensations were still sharply defined, the components P1 and $\mathrm{T}$ were barely discernible as virtually uncondensed masses of material. Again, no molecular emissions were detected in the 2220-3280 $\AA$ region.

The HST monitoring of the comet continued throughout the months of May-July 1994, one of the highlights of this period being the spectral detection of a strong outburst of $\mathrm{Mg}^{+}$on July 14 (Weaver et al. 1995, Feldman et al. 1996; the continuum outburst observed $\sim 18$ mins after the $\mathrm{Mg}^{+}$outburst was probably due to the passage of a faint star through the spectrograph aperture, according to the latter reference). The absence of any neutral molecular emissions in the spectrum between 2220 and $3280 \AA$ was again confirmed. The images show that the central regions of the condensations, a few seconds of arc across, remained spherically symmetric until one week or so prior to impact, at which time they began to grow strikingly elongated along the direction of the train.

In the meantime, ground-based observations-interrupted in late July 1993 because of the comet's approaching conjunction with the Sun-resumed in early December 1993 and continued during 1994 until impact. The condensation P2 was observed extensively and as late as July 14 at the European Southern Observatory at La Silla and July 19 at the Mauna Kea Observatory. Ground-based observations of P1 apparently terminated in late March. The condensation Q2 may have been sighted on only a few occasions and was measured perhaps just once. On May 7, 1994 Jewitt \& Trentham (1994) detected a companion to the condensation G, 5.1 arcsec to the northeast of it. The existence of this companion G2 was confirmed on the HST images taken on May 17, by which time its separation distance increased to 5.9 arcsec (Noll \& Sinith 1994). On the other hand, its identification with a faint object located 4.1 arcsec to the north-northeast of $G$ on the HST images from March 29 is doubtful.

\subsection{The dust trails}

Observations of the two trails are very limited, compared with the extensive amount of information available on the nuclear train. Almost all the published data refer to the early post-discovery period, late March through late May 1993. Their nearly complete 

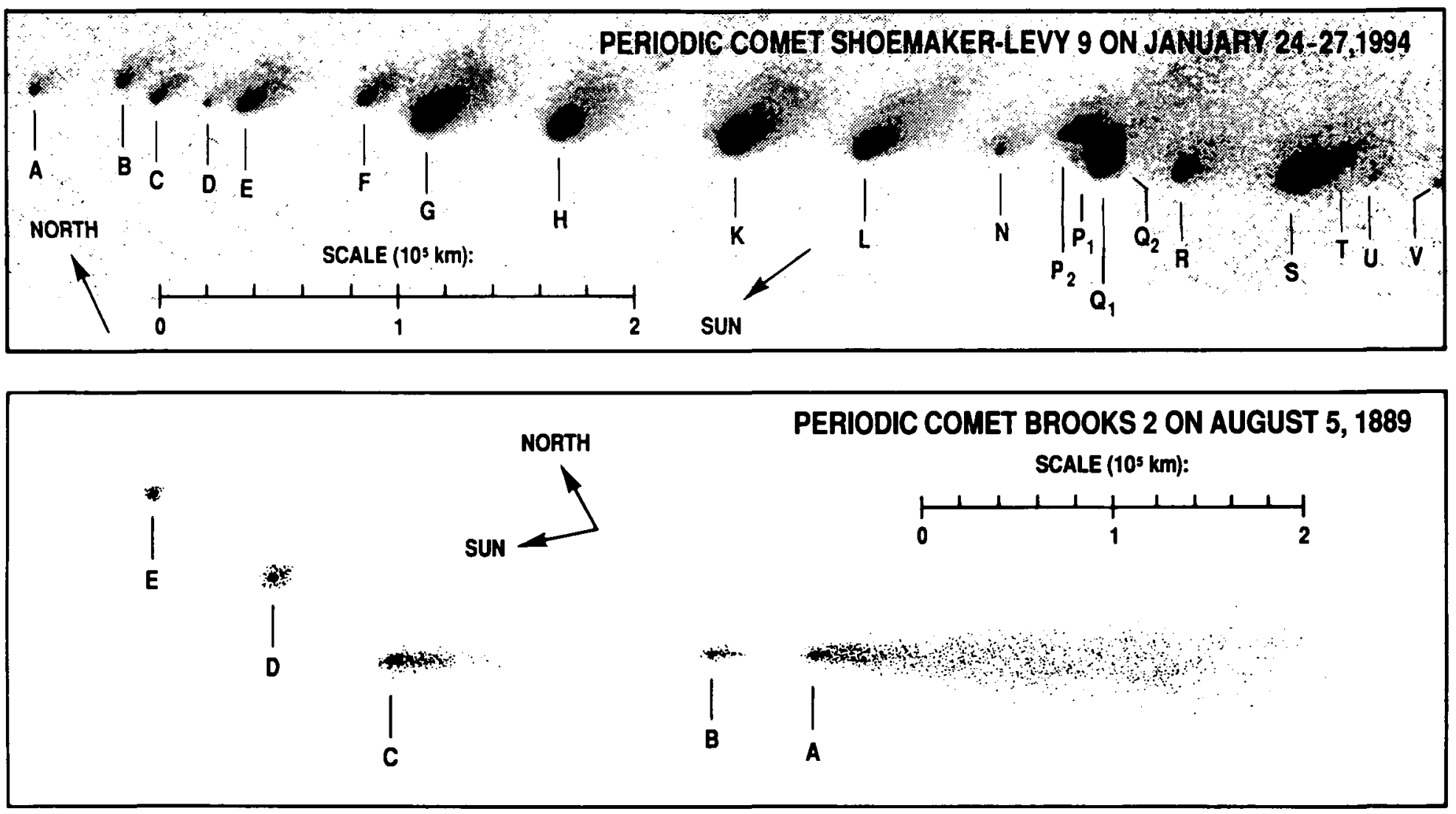

FigurE 1. Comparison of the appearance of periodic comets Shoemaker-Levy 9 and Brooks 2. Top: Mosaic image of the nuclear train (except for the condensation W) and the tails of Comet Shoemaker-Levy 9 taken with the Wide Field Planetary Camera 2 of the Hubble Space Telescope on January 24-27, 1994. The 20 visible condensations are identified in the commonly used notation introduced by Sekanina et al. (1994) and later expanded to accommodate the additional condensations. The projected linear scale at the comet and the directions of the north and the Sun are shown. (Adapted from an image provided by courtesy of H. A. Weaver and T. E. Smith, Space Telescope Science Institute.) Bottom: Drawing of P/Brooks 2 made by E. E. Barnard (1889) and based on his visual observation with the 91-cm equatorial of the Lick Observatory on August 5, 1889. The notation used for the five condensations is that of Barnard. The scale and the orientation of the drawing are also shown. 
list is presented in Table 2 of Paper 1 . The west-southwestern trail was perfectly aligned with the nuclear train and its southern boundary appeared to be marginally sharper than that of the east-northeastern trail. The two trails made an angle of $176^{\circ}$ with each other, with an uncertainty of about $\pm 2^{\circ}$. No morphological features that could possibly suggest the presence of faint condensations were ever reported in either trail, but it appears that not enough effort has been made with this goal in mind. To the north, the trails blended into the structureless sector of material. From the descriptions based on 10 low-resolution images, the length of the east-northeastern branch was, on the average, 0.63 the length of the west-southwestern branch. The maximum lengths were reported by Scotti (1993) on March 30, 1993: 10.4 arcmin at a position angle of $260^{\circ}$ for the west-southwestern branch and $\sim 6.2$ arcmin at $75^{\circ}$ for the east-northeastern branch, measured from the train's midpoint. Computer processed images show, however, no clear difference between the lengths of the two branches (Scotti \& Metcalfe 1995). The only 1994 observations of the trails that I am aware of were reported by Lehký (1994) and by Scotti \& Metcalfe (1995), both during February. The trails were fading rapidly with time and their expansion was consistent, according to Scotti \& Metcalfe, with the assumption of no evaporation and no further production of dust material.

\subsection{The tails}

The tails associated with the condensations were observed virtually at all times between discovery and collision. Their orientations and lengths derived from the comet's groundbased images taken in the early period after discovery were summarized in Table 2 of Paper 1. The tails were reported to point at this time at the position angles between $280^{\circ}$ and $300^{\circ}$, making an angle of $\sim 30^{\circ}$ with the nuclear train. Their lengths, depending on observing conditions and on the intrumental resolution and sensitivity, the exposure time, and the spectral window, were found to be up to $\sim 80$ arcsec. Additional tail observations were made by Scotti \& Metcalfe (1995) in both 1993 and 1994 and by R. M. West et al. (1995) shortly before impact. These results are discussed in Sec. 4.4.

The tails are displayed prominently on most of the HST images obtained in 1994, an example of which, from late January, is reproduced in Fig. 1. Each condensation had its own tail, whose length and degree of prominence clearly correlated with the "parent" condensation's brightness. The tails of the major condensations, such as $\mathrm{G}$ or $\mathrm{K}$, are seen to have extended at this time all the way to the edge of the field and their lengths must have greatly exceeded $25 \operatorname{arcsec}$, or $100,000 \mathrm{~km}$ in projection onto the sky plane.

The apparent breadth a few seconds of arc from the condensation is estimated at $\sim 6-7$ arcsec for the brightest tails, but only at $\sim 2$ arcsec or so for the fainter ones, corresponding to projected linear widths of 8000 to $25,000 \mathrm{~km}$. There is evidence that the tails of some of the condensations (such as $\mathrm{E}, \mathrm{H}$, and S) broadened more significantly with distance from the train than did the tails of other condensations (such as $\mathrm{C}, \mathrm{K}$, and L). There also is an indication that the angle between the directions of the train and the tails might have been getting smaller with time.

\subsection{Comparison with other tidally split comets}

Even though the appearance of comet Shoemaker-Levy 9 was unquestionably unique among observed comets, certain similarities, however remote, can be found with two other tidally disrupted comets, P/Brooks $2(1889 \mathrm{~V})$ and the sungrazer 1882 II. The recognition of such similarities is of the essence in the context of establishing the diagnostics of tidally split objects and their observable characteristics.

During its approach to Jupiter on July 21, 1886, the jovicentric orbit of P/Brooks 2 was slightly hyperbolic (Sekanina \& Yeomans 1985) and the comet's post-encounter orbital 
evolution was very different from that of Shoemaker-Levy 9. After passing two planet's radii from the center of Jupiter, $\mathrm{P} /$ Brooks 2 settled in a new heliocentric orbit, whose perihelion distance was $1.95 \mathrm{AU}$. The comet was discovered about three years later and was 1.16 AU from the Earth and 2.01 AU from the Sun and approaching the perihelion, when Barnard (1889) made the object's drawing on August 5, 1889, shown in Fig. 1. Two of the morphological features recognized in comet Shoemaker-Levy 9 are apparent on this drawing: (i) the nuclear train, consisting of the condensations $\mathrm{A}, \mathrm{B}$, and $\mathrm{C}$ and (ii) the tails, which in this case were aligned with the train. The condensations $\mathrm{D}$ and $\mathrm{E}$, either of which was seen on only two occasions, represent a pair of "anomalous", off-train components analogous to Shoemaker-Levy 9's condensations B, F, N, etc. In addition, on one night Barnard $(1889,1890)$ detected four other faint companions to the south of the train and more distant from $\mathrm{A}$ than was $\mathrm{E}$, but all of them remained unconfirmed, as did a companion reported by Renz (1889) on another day. Barnard (1890) suspected that at least some of these objects may have in fact been faint, unrecorded nebulae.

There was no evidence for wings extending from the condensations in either direction or for a sector of diffuse material spreading to either side of the train. However, Weiss (1889) reported the detection of a nebulous sheath encompassing the condensations A and $B$, while Barnard (1890) remarked on the absence of any such nebulosity during his observations on the same dates.

The relatively straightforward, empirically inferred parallelism between some of the morphological properties of comets Shoemaker-Levy 9 and Brooks 2 is found to be rather encouraging and appealing. Yet, the differences between the two objects and between their dynamical evolutions following the close encounter with Jupiter are significant enough that caution should be exercised not to overinterpret the similarities.

The nuclear region of the sungrazing comet 1882 II was observed to consist of at least five condensations after perihelion. They lined up in the orbital plane in a direction that, after correcting for effects of foreshortening, was lagging the Sun-comet line by more than $20^{\circ}$ in the early post-perihelion period, but gradually less with time, until the two directions coincided some 5-6 months later, at the time of the final observations (Kreutz 1888). The brightness of the individual components varied with time, but the second and the third condensations from the train's sunward end were consistently the most prominent ones. An elongated nebulous sheath of material was enclosing the entire train. When last measured, about 150 days after perihelion, the sheath was almost 3 arcmin long, which is equivalent to a projected extent of more than $300,000 \mathrm{~km}$. It is conceivable that the far regions of the sheath would have eventually evolved into wings similar to those displayed by comet Shoemaker-Levy 9 , if they were sufficiently bright to have remained under observation still longer.

\section{Implications for modelling comet Shoemaker-Levy 9}

Before turning to the discussion of the various models proposed for comet ShoemakerLevy 9, I list a few critical issues that represent excellent test criteria and are therefore to be addressed first. The merit of a paradigm depends primarily on the degree of its conformity with observations used in the course of its formulation, on the plausibility of the assumptions employed, and obviously very much on the success of any verifiable predictions that it might offer.

\subsection{Morphological test criteria}

From the descriptions of the comet's appearance in Sec. 3, two criteria are identified for testing a model: (i) it should explain quantitatively the four classes of morpho- 
logical features detected (the nuclear train, trails, tails, and the sector of material) and their evolution and properties; and (ii) it should be conceptually consistent with unambiguous, model-independent conclusions based on direct observational evidence. Each model should also be judged in a broader context, in such terms as the object's implied long-term dynamical stability, the plausibility of postulated properties, and the paradigm's compatibility with current views on the physical behavior of comets.

The aspects of the problem that are considered the most significant are individually discussed below. The first point to make is that each of the four classes of morphological features presents an important piece of observational evidence. As such, these diverse features should either be consistently interpreted as different manifestations of the same process and as individual products of the parent object in the context of the tidal breakup and its consequences; or else be explained by a credible, independent hypothesis that should account for their existence, appearance, and temporal evolution. A model that leaves some of this morphology unexplained, is inevitably incomplete and tentative and its merits and the conclusions to which it leads should be viewed with caution and some skepticism.

The second point to make is that although the nuclear train should not be singled out as the only target of modelling efforts, its morphology offers more information than do the other features and its analysis should be of top priority. Some of the train's properties are diagnostically so critical that they deserve special attention.

\subsection{The train's orientation}

Temporal variations in the train's orientation are among the dynamical characteristics of comet Shoemaker-Levy 9 that have been determined with very high accuracy and are of fundamental importance from the standpoint of modelling. The dependence of the position angle of the nuclear train on the conditions at the time of tidal disruption was investigated in Paper 1. The orbital calculations showed conclusively that for a given trajectory of the comet and a fixed time of breakup, no measurable effect on the train's orientation could be generated by variations, within physically meaningful ranges, in the initial radial distances of the fragments from Jupiter (which represent the dimensions of the parent nucleus) or in their orbital velocities (which simulate any effects in the rotational and/or translational momentum), or some combination thereof. The only parameter that demonstrably affects the train orientation in a systematic manner is the model-independent time of dynamical separation, also called the effective time of breakup.

In practice, the train's position angles derived from astrometric observations were found to depend slightly, but measurably, on the selection of the condensations. For the sake of uniformity, it was necessary to select a "standard" set of condensations. Since the origin of the group of anomalous condensations ( $B, F, J, M, N$, etc.) was suspect, its members were the first to be excluded from any such standard set. The selection was essentially dictated by a balance between two somewhat contradictory requirements: (i) by the need to employ the largest possible number of observations and (ii) by a condition that the position angles calculated from the standard set be representative of the "intrinsic" orientation of the train as a whole. Since most observers measured only brighter condensations, the first point implies the need to employ as few condensations as possible. By contrast, the second point requires a large number. After much experimentation, the standard set was defined in Paper 1 by eight condensationsE, G, H, K, L, Q (later Q1), S, and W. Comparisons with other sets showed that, except when the anomalous condensations were much involved, the position angles differed at most by a few hundredths of a degree. This is only a small fraction of the typical uncertainty involved in the position-angle determinations, which was about \pm 0.1 for 


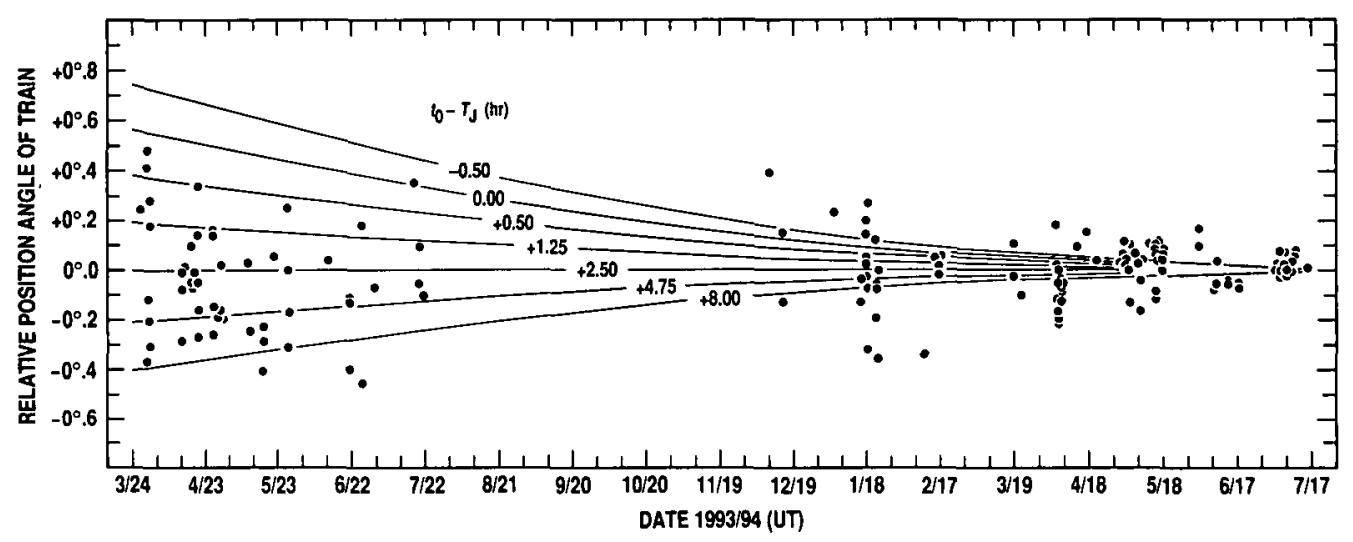

FIguRE 2. The temporal distribution of residuals of the nuclear train's position angle as a function of the assumed effective time of tidal disruption, $t_{0}$. The dots represent the 144 data points, determined by fitting astrometric observations of the standard set's eight condensations $\mathrm{E}$ through $\mathrm{W}$. The curves show the variations in the calculated position angle for seven different effective times of breakup measured relative to the time of perijove, $T_{\mathrm{J}}=1992$ July $7.860364 \mathrm{TDB}$. For the optimized solution, $t_{0}-T_{\mathrm{J}}=+2.5$ hours, the calculated variations are represented by a straight line. (After Sekanina et al. 1995.)

high-resolution images (telescopes of $\sim 1.5$-meter aperture or larger) and some \pm 0.3 or so for images of lower resolution (telescopes of $\sim 1$-meter aperture or smaller).

Although relatively subtle, the deviations of the nuclear train orientation from the values predicted for perijove were found in Paper 1 to be sufficiently pronounced that the effective time of breakup, $t_{0}$, could be determined, with an estimated error of \pm 0.5 hour or so, from observations made primarily in the early post-discovery period of time. The time $t_{0}$, which describes the completion of a post-breakup collisional redistribution of the debris rather than the initiation of tidal fracture, was calculated in Paper 1 to equal 2.2 hours after perijove, based on a total of 42 astrometric positions from 1993. A new result based on the recently expanded database, including all 144 relevant observations from 1993-1994 (Sekanina et al. 1995), essentially confirms the solution derived from the smaller sample; the statistically best estimate is now 2.5 hours, again with an estimated error of \pm 0.5 hour. The distribution of the residuals in Fig. 2 shows that the 1994 observations, while entirely consistent with the 1993 ones, fail to improve the solution significantly because of a dramatic decrease in the sensitivity. The detailed interpretation of the effective breakup time is an issue that each model is to settle within its own framework. I will return to this problem in Sec. 5 .

\subsection{Secondary fragmentation}

The next fundamental issue is that of the origin and the nature of the off-train, anomalous condensations. This point, too, was addressed in Paper 1 on the example of the motion of the component $\mathrm{P}$ relative to $\mathrm{Q}$. The 12 relative positions between late March 1993 and mid-January 1994 could be satisfied with a mean residual of \pm 0.28 arcsec, if $P$ separated from $Q$ in mid-November 1992 (with an uncertainty of a few weeks) at a relative velocity of $0.9 \mathrm{~m} \mathrm{~s}^{-1}$. Evidence for similar events of secondary fragmentation became ubiquitous during 1994. Two examples are reproduced in Figs. 3 and 4, both based on the results of a work in progress (Sekanina et al. 1995). Figure 3 displays an updated solution to the motion of $\mathrm{P} 2$ relative to $\mathrm{Q}$, which satisfies 74 observations between March 27, 1993 and July 19,1994 with a mean residual of \pm 0.29 arcsec. The breakup was found to have occurred on 1992 Dec. $29 \pm 9$ days with a separation velocity of $1.14 \pm 0.08 \mathrm{~m} \mathrm{~s}^{-1}$. 


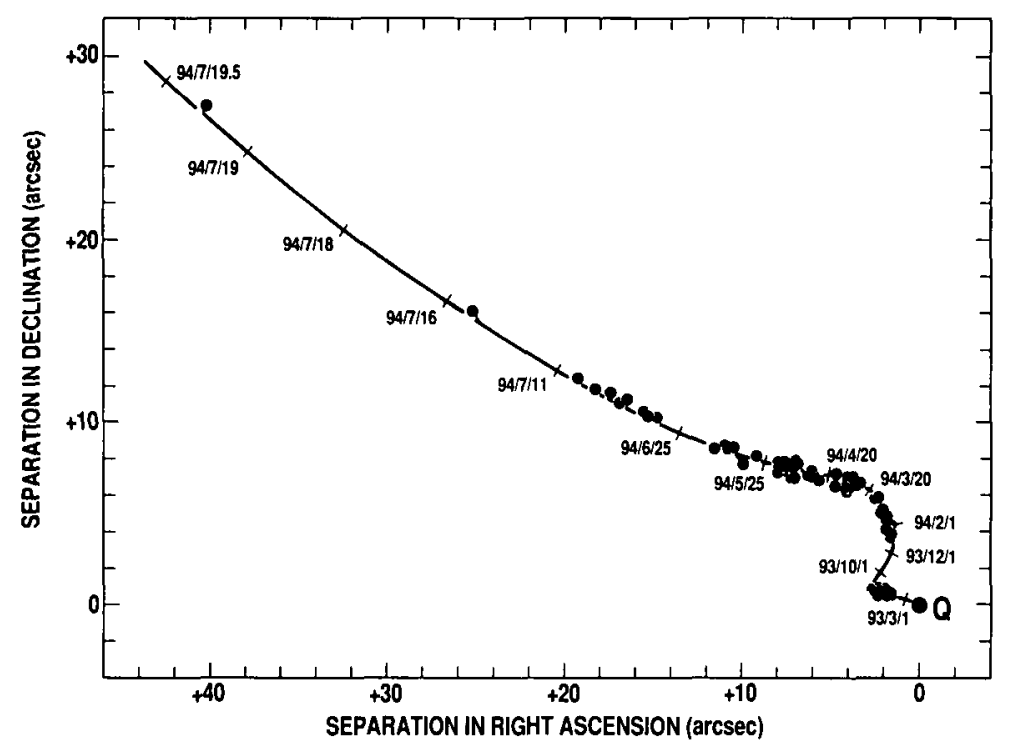

Figure 3. Projected motion of the component $P 2$ relative to $Q$, based on 74 observations between March 27, 1993 and July 19, 1994 and interpreted as due to a splitting of the two condensations on Dec. 29, 1992 with a relative velocity of $1.14 \mathrm{~m} \mathrm{~s}^{-1}$. The dots are the observations and the curve shows the optimized dynamical solution. (After Sekanina et al. 1995.)

Figure 4 displays the motion of $\mathrm{Q} 2$ relative to $\mathrm{Q} 1$, based on very accurate measurements from the HST images. The eight positions cover the period of time from July 1, 1993, when the two components were just 0.3 arcsec apart, to July 20, 1994. The solution, leaving a mean residual of $\pm 0.029 \operatorname{arcsec}(!)$, yields 1993 Apr. $12 \pm 8$ days for the date of splitting and $0.32 \pm 0.02 \mathrm{~m} \mathrm{~s}^{-1}$ for the separation velocity.

The fact that the comet's fragmentation continued as a sequence of discrete events for a considerable time after the 1992 grazing encounter with Jupiter has enormous ramifications. First of all, secondary fragmentation was positively nontidal in nature. Next, for all practical purposes it is certain that all of the off-train condensations were products of this process, so that the number of major components split off from the parent comet by tidal fracture was not 21 , but most probably 10-12. The preliminary results also indicate no need to introduce differential accelerations in the motions of the off-train condensations, although much work still remains to be completed. The apparent absence of such accelerations lends support to tentative conclusions that neither effects of solar radiation pressure nor nongravitational effects have been detected, and that these condensations were neither loose assemblages of small-sized particulate material nor did they display any activity.

Secondary fragmentation will unquestionably stay in the forefront of attention for some time to come. A plausible physical model for Shoemaker-Levy 9 must account for this sequence of discrete events, most of which appear to have taken place between early July 1992 and the beginning of 1993, when the nuclear train was receding from Jupiter, and also explain the observed relative velocities involved, of up to at least $1 \mathrm{~m} \mathrm{~s}^{-1}$.

\subsection{Tail orientation and morphology: Relation to the problem of activity}

The problem of Shoemaker-Levy 9's outgassing activity, especially after discovery, has been a hotly debated issue. With the exception of the brief appearance of $\mathrm{Mg}^{+}$shortly before impact (as already mentioned in Sec. 3.2), no emission was ever detected spectroscopically. The unsuccessful search for the hydroxyl radical with the HST's Faint Object 


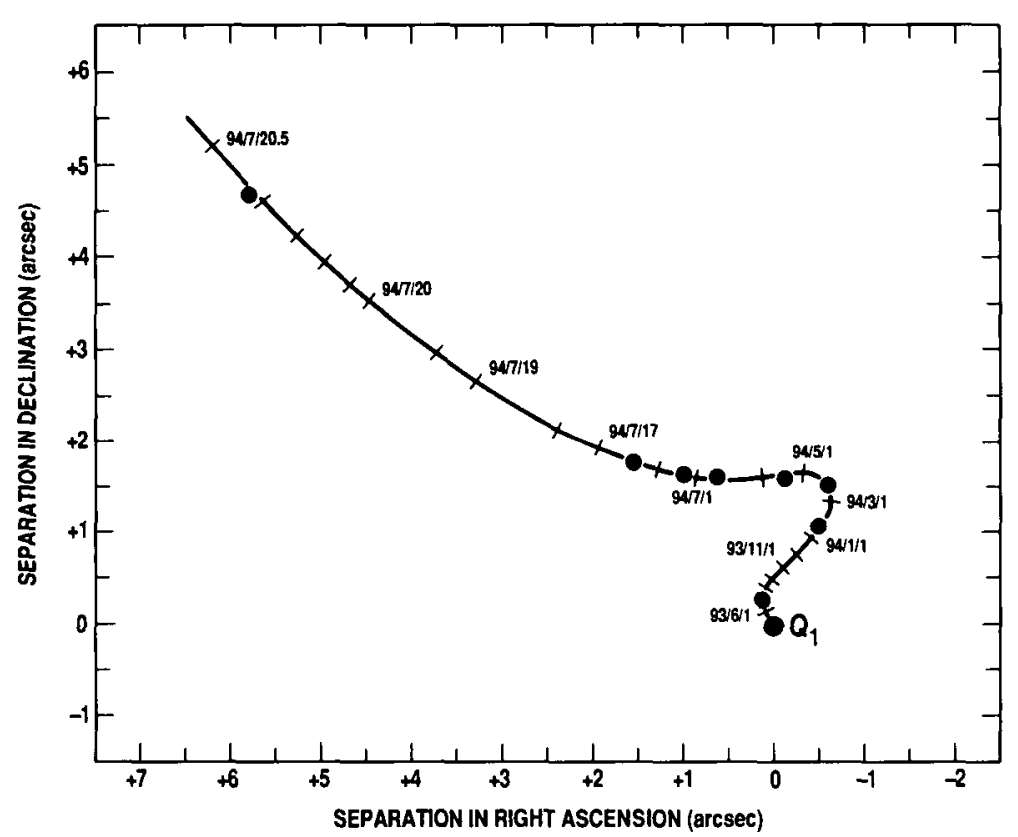

FIGURE 4. Projected motion of the component Q2 relative to Q1, based on eight HST observations made between July 1, 1993 and July 20, 1994 and interpreted to result from a splitting of the two condensations on Apr. 12, 1993 with a relative velocity of $0.32 \mathrm{~m} \mathrm{~s}^{-1}$. The dots are the observations and the curve shows the optimized dynamical solution. (After Sekanina et al. 1995.)

Spectrograph and from the ground yielded a $3 \sigma$ upper limit on the water production rate of 30 to $60 \mathrm{~kg} \mathrm{~s}^{-1}$ (Weaver et al. 1995; quoted here is the range of corrected values).

Indirect information on the object's possible activity is provided by the orientation and morphology of the dust tails. It was stated in Paper 1 that the orientation of the tails observed in March 1993, shortly after the comet's discovery, essentially supports the conclusion that they consisted of particulate material that had been released during the tidal breakup in early July 1992 and subsequently subjected to effects of solar radiation pressure. The results of R. M. West et al.'s (1995) careful study of the tails of the fragments $\mathrm{G}$ and $\mathrm{K}$ in the period of July 1-15, 1994 are also generally consistent with this conclusion. The new preliminary results of our work (Sekanina et al. 1995), which is still continuing, indicate that, using an updated set of orbital elements for the fragments, we have apparently detected small but systematic deviations of the tails' reported position angles from the tidal-breakup synchrone. The dependence of the tail orientation on the time of particle release is plotted in Fig. 5. The observed tail orientations are from four sources: (i) most of the data in March 1993 are from Table 2 of Paper 1; (ii) the majority of the points before July 1994 are preliminary values for the fragment G by Scotti \& Metcalfe (1995), with an estimated uncertainty of several degrees; (iii) two points are the author's estimates on available HST prints (for six fragments in each case); and (iv) the points in July 1994 are averages of R. M. West et al.'s (1995) results for the fragments $\mathrm{G}$ and $\mathrm{K}$ at $15,000 \mathrm{~km}$ from the condensation. It is evident from Fig. 5 that the synchrones corresponding to times of release in September-November 1992 are formally more consistent with the observed orientations than the synchrone of early July 1992. Because of uncertainties involved in the measured position angles and the poor resolution due to the "crowding" of the synchrones, all one can conclude at this time is that the 


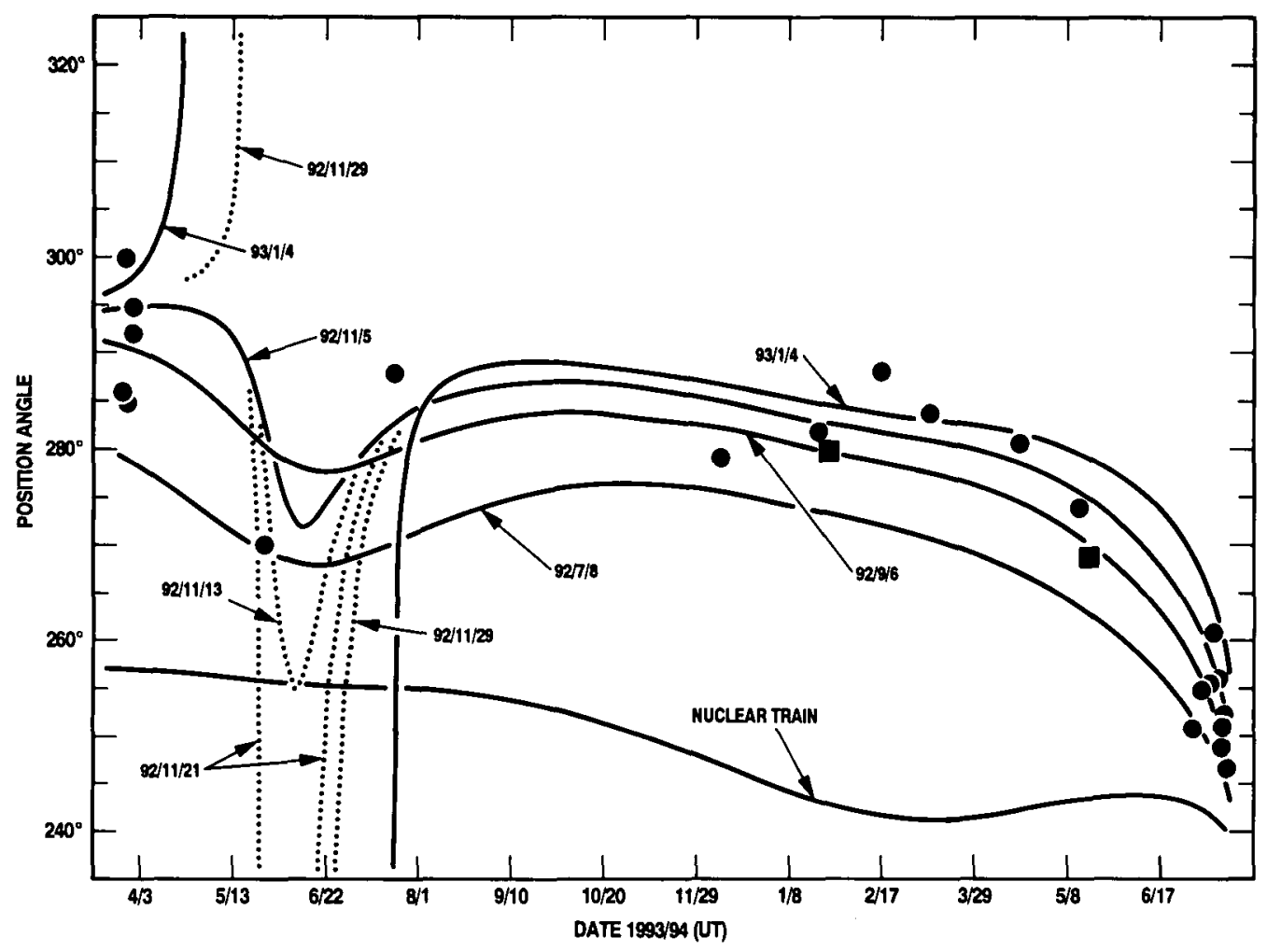

FIGURE 5. The tail orientation as a function of the assumed time of release of particulate material. The position angles measured on ground-based images are plotted as circles; those estimated by the author from two HST prints, as squares. Most of the March 1993 data are from Table 2 of Paper 1; the majority of the points before July 1994 are preliminary values for the fragment G by Scotti \& Metcalfe (1995); and those from July 1994 are averages of R. M. West et al. (1995) results for the fragments $G$ and $K$ at $15,000 \mathrm{~km}$ from the condensation. The synchrones for particles released in mid- through late November 1992 became nearly aligned with the comet-Earth line during May and June 1993, resulting in a rapid variation in the position angle (dotted curves). The nuclear-train orientation is plotted for comparison. (After Sekanina et al. 1995.)

observed tails consisted of dust particles released most probably during the period of time between early July and the end of 1992. Available evidence does not make it possible to decide (i) whether the release of the particulates was continuous or proceeded in a sequence of discrete events and (ii) whether or not it was outgassing-driven.

From the temporal coincidence it is tempting to associate the tail formation with both the tidal breakup and the events of secondary fragmentation. However, unless more is learnt about Shoemaker-Levy 9's tails in the future, the proposed relationship with the secondary-fragmentation events will necessarily remain speculative.

It was argued in Paper 1 that if the fragments were active, they would have displayed tails to the east-southeast from the condensations during certain periods of time (spanning many months), contrary to the observations. In order to address a counterargument (Weaver et al. 1995) that such tails may not have been detectable, if the dust production rates were very low, it clearly is desirable to obtain at least crude quantitative estimates for critical dust production rates. Although this issue is not investigated here systematically, two examples shown below are sufficient to illustrate approximate limits that such considerations imply. In both examples the particle size distribution function is assumed 
to vary inversely as the fourth power of the particle size-a reasonable approximation supported by results on a number of comets, the visual geometric albedo of the dust is taken to be equal to 0.04 , and the total dust production rate is understood to refer to particles whose sizes range from $0.1 \mu \mathrm{m}$ to $1 \mathrm{~cm}$. For the assumed size distribution, the results are relatively insensitive to the choice of the size limits; increasing, for example, the upper limit from $1 \mathrm{~cm}$ to $1 \mathrm{~m}$ would increase the production rate by a factor of only 1.4. In the first of the two cases, I assume dust particles ejected in early January 1993. In late May 1993 some of these ejecta would be located in a tail about 15 arcsec away from the parent fragment at a position angle of $\sim 110^{\circ}$. A conservative assumption of a tail detection threshold of, say, $\sim 23 \mathrm{mag} / \operatorname{arcsec}^{2}$ in the visual passband on ground-based images implies an upper limit on the dust production rate of $\sim 5 \mathrm{~kg} \mathrm{~s}^{-1}$, equal to the steady-state rate derived by Weaver et al. (1995) from the observed spatial profile of the brighter condensations. In the second case, I assume ejections near March 20, 1994. On the comet's image taken with the HST on May 17, 1994 (Fig. 1 of Weaver et al. 1995), a tail consisting of these ejecta should be at a position angle of $\sim 103^{\circ}$. At $\sim 5 \operatorname{arcsec}$ from the parent fragment, a surface brightness of 2 ADU per WF pixel ${ }^{2}$ in a red passband, which should have easily been detectable (Weaver 1995), implies a conservative upper limit of merely $0.5 \mathrm{~kg} \mathrm{~s}^{-1}$, one order of magnitude lower than the limits quoted above.

The conclusion that the tails consisted of dust released exclusively (or nearly so) in the second half of 1992, sets tight constraints on particle sizes and velocities. First of all, solar radiation pressure accelerations on dust grains in the tails are found to have been extremely low. For example, on the July 1994 images taken by R. M. West et al. (1995) a typical acceleration ratio of radiation pressure to solar gravity on particulates located $15,000 \mathrm{~km}$ from the parent fragment amounted to 0.00005 , if they were released in early July 1992 , or to 0.00020 , if in late 1992 . These are pebble-sized objects, with typical diameters between 1 and $10 \mathrm{~cm}$, depending on their bulk density. The width of the tails is determined by limits to the particle velocity distribution in the plane normal to the orbital motion; from the projected linear widths in late January 1994 (Sec. 3.4) and the age of the particulates at the time $(\sim 13-19$ months), one finds characteristic velocities in the range from 0.1 to $0.4 \mathrm{~m} \mathrm{~s}^{-1}$, comparable with the separation velocities of the products of secondary fragmentation (Sec. 4.3). And since each tail is an obvious outgrowth of its parent condensation, particles in the latter must have had even larger sizes and lower velocities.

An independent argument against any meaningful level of activity of Shoemaker-Levy 9 is the absence of detection of any diagnostic morphology in the condensations. If a comet ejects dust-especially as far from the Sun as 5-6 AU-it does so not from the entire nuclear surface, not even from its entire sunlit side, but from a discrete, suddenly activated spot. As the ejected material propagates through the comet's atmosphere, it forms some kind of a gradually expanding feature, whose coma-residence time scale is usually a few weeks at heliocentric distances comparable with that of Jupiter. One such example is $29 \mathrm{P} /$ Schwassmann-Wachmann 1. Another, appearing quite recently, is Hale-Bopp (1995 01). Absolutely no such feature was ever observed in Shoemaker-Levy 9. Even the bright "spur" (Weaver 1994) in the condensation S would not do, as it has an explanation that requires no activity (Sekanina 1995a,b).

\section{Models for the splitting of comet Shoemaker-Levy 9}

All published models for comet Shoemaker-Levy 9 are based on the assumption that the Jovian tidal forces were either entirely, or primarily, responsible for the comet's breakup. 
This notion is supported by the orbital calculations, which show that the spatial positions of the various condensations, extrapolated from their absolute astrometric observations in 1993-1994 back in time, essentially coincided with one another at the time of closest approach to Jupiter on 7 July 1992 (Yeomans \& Chodas 1994), the most satisfactory correspondence being indicated by the on-train condensations.

\subsection{Classification of the models and a historical perspective}

There are basically two critical discriminants for the various models: (i) the gross nucleus structure and morphology of the progenitor comet and (ii) the role of particle-particle collisions. The first discriminant divides the existing models of the parent nucleus into two groups: either a strengthless "rubble pile," an agglomerate of smaller building blocks of material held together entirely by self-gravity; or a discrete mass of solids-whether or not of aggregate structure-possessing a definite cohesive strength. The second discriminant separates the models that account for effects of collisions accompanying the breakup from the models that treat the problem as that of a collisionless process.

Diverse morphology of the proposed models for Shoemaker-Levy 9 is reminiscent of an old controversy between the $i c y$ conglomerate nucleus of Whipple $(1950,1951)$ and the sand bank model, which went on for decades and was thought-prematurely, as it now appears-to have been settled in favor of Whipple's concept by the images of Halley's nucleus returned by the Giotto and Vega spacecraft. As pointed out by Whipple (1961, 1963), the sand bank paradigm has been around, in one form or another, for a century or so. Its extreme variation-a diffuse, loose swarm of particles-was advocated by Lyttleton (1953), but in other versions the cometary nucleus was envisaged as a much more compact agglomerate. For example, Vorontsov-Velyaminov (1946) maintained that the nucleus of Halley's comet is $\sim 30 \mathrm{~km}$ in diameter and consists of a cluster of meteoric blocks, each $\sim 150$ meters across, which are nearly in contact. Schatzman (1953) concluded that a compact sand bank assemblage could collapse under certain circumstances, if protected from dispersive forces, but he cautioned that in relevant scenarios the process might be too slow. Whipple $(1961,1963)$ showed that the compact sand bank model encounters most of the difficulties of the diffuse model and pointed out that gravitational coherence alone is unlikely to keep the nucleus intact over extended periods of time. While one may argue that comet Shoemaker-Levy 9 is an exception to the rule, an ad hoc postulate of this kind is questionable and should be viewed with skepticism.

\subsection{Strengthless agglomerate models: The pros and cons}

At least two models describe the nucleus of comet Shoemaker-Levy 9 as a strengthless agglomerate of subkilometer-sized cometesimals (Asphaug \& Benz 1994, 1996, Solem 1994, 1995). Both are concerned only with the nuclear train and the main difference between them is that Solem's model accounts for effects of particle-particle collisions. A third model, briefly described by Rettig et al. (1994), was at the time of this writing still in the process of development; in some of its versions a nonzero mechanical strength among the assumed $\sim 50$-meter-sized cometesimals is considered (Mumma 1995). All distances in the strengthless models scale with simple similarity and the results depend on the bulk density of the assemblage, as can readily be illustrated on a spherical body, whose radius is $R$, bulk density $\rho$, and gravitational pressure at its center $P_{\mathrm{c}}$. The gravitational attraction between its two hemispheres is $\frac{1}{2} \pi R^{2} P_{\mathrm{c}}$, while the net tidal force from Jupiter, of radius $R_{0}$ and density $\rho_{0}$, amounts to $\pi R^{2} P_{\mathrm{c}}\left(\rho_{0} / \rho\right)\left(R_{0} / \Delta\right)^{3}$ at a distance $\Delta$ from the planet. The necessary condition for a hemispheric separation isindependent 
of the radius $R$ and is given by the following expression (Aggarwal \& Oberbeck 1974, Dobrovolskis 1990)

$$
\frac{\rho}{\rho_{0}}\left(\frac{\Delta}{R_{0}}\right)^{3}<2 .
$$

For Shoemaker-Levy 9 one finds $\rho<1.1 \mathrm{~g} \mathrm{~cm}^{3}$, probably a soft limit, since the breakup is likely to have begun before perijove (cf. also the investigations by Boss 1994 and by Greenberg et al. 1995). A more meaningful constraint is offered by $\mathrm{P} /$ Brooks 2, which approached Jupiter to 2 Jovian radii, so $\rho<0.34 \mathrm{~g} \mathrm{~cm}^{3}$ (Sekanina \& Yeomans 1985).

An attractive attribute of the self-gravitating strengthless compact agglomerate models is their apparently successful simulation of the progenitor's tidal disruption into a fairly small number (between a few and two dozen or so) of discrete clumps of debris, if the bulk density of the cometesimals is confined to a narrow interval of plausible values. The reason for this unexpected result is that shortly after passing through perijove the cometesimals begin to reassemble gravitationally, but because of the considerable tidal elongation of the cloud at that time, the coagulation proceeds only locally, if the bulk density $\rho$ is, in the particular case of Shoemaker-Levy 9 , close to $0.5 \mathrm{~g} \mathrm{~cm}^{3}$.

Unfortunately, the Asphaug-Benz-Solem paradigm of a strengthless nucleus has a number of grave conceptual weaknesses. Besides the fact that the complete absence of material cohesion is unphysical (Greenberg et al. 1995), two of the most serious shortcomings of these proposed strengthless models are (i) the assumption of equal-sized cometesimals that make up the nucleus and (ii) their "typical" chosen size of more than 100 meters across. Attempts to match the comet's brightness, reported by visual observers to have attained a total magnitude of 12-13 shortly after discovery, and thereby to accommodate the object's enormous cross-sectional area, run into unsurmountable problems. For example, with the parent nucleus $1.5 \mathrm{~km}$ and each cometesimal $0.15 \mathrm{~km}$ in diameter, typical of the Asphaug-Benz-Solem model, one infers from it-ceven in the absence of inevitable mutual particle shadowing - that the comet should have had an apparent visual magnitude of $22-23$ for a plausible geometric albedo of $<10$ percent, tacitly implying that the comet should not have been discovered with the Palomar 0.46-cm Schmidt telescope! Even with an entirely unrealistic albedo of unity, the comet still could only have been of magnitude 19. On the other hand, a constant size of the cometesimals that would satisfy the conditions for the parent comet's diameter and the observed brightness is found to be a small fraction of $1 \mathrm{~cm}$, or more than four orders of magnitude smaller than the size used in the models. The only possible way to avoid the contradiction is to allow the cometesimals to possess a broad size (and mass) distribution, as considered by Sekanina et al. (1994). Since the self-gravity field of a cloud of particles depends on their masses, the introduction of a broad mass distribution has to have a dramatic effect on the cloud's stability, as well as on the tidal disruption and the subsequent clumping of the cometesimals. This mass distribution effect can in no way be predicted or estimated from the results of the proposed equal-size strengthless models.

The strengthless agglomerate models also experience difficulties with explanations of two well documented phenomena. One is secondary fragmentation (Sec. 4.3), the other is the sharp contrast, shortly before crash, between Jovian-gravity driven, progressive stretching of each condensation's dust coma and the complete absence of this effect in its innermost, brightest part, which retained the appearance of an unresolved dot (cf. the last HST frame of Q1 and Q2 in Weaver et al.'s 1995 Fig. 2). A supporter of the strengthless agglomerate model now has a choice: he can assume that, following the comet's 1992 tidal disruption and subsequent clumping, the central fragments in the condensations became gravitationally either stable or quasi-stable. If they were stable, they could not disrupt 
again months later and secondary fragmentation remains unexplained. If they were quasistable, secondary fragmentation is possible, but then the central fragments should have stretched during their 1994 return to Jupiter just as did the surrounding dust clouds, because gravitational stretching is mass independent, except in stable configurations, which are explicitly ruled out. The lack of progressive stretching of the central objects in the condensations now remains unexplained.

While the Asphaug-Benz-Solem strengthless assemblages are not defined in sufficient detail to choose between the stable and quasi-stable scenarios, the above example shows that either scenario can selectively be manipulated to explain one phenomenon at a time, but not to offer conceptually consistent interpretations of two or more phenomena. From Asphaug \& Benz's $(1994,1996)$ analysis it appears that the clumps must have been relatively stable, if self-gravity began to prevail over the Jovian tides as early as several hours after perijove. However, the perception of the Asphaug-Benz paradigm in the comet community is clearly exemplified by Weissman's (1994) grossly incorrect predictions for the impact phenomena, based on this model. The motivation for such misleading conclusions is obvious: loose assemblages of hundred-meter-sized cometesimals should indeed have disintegrated into debris at high Jovian altitudes and we should have witnessed a cosmic fizzle. Whereas observations do provide substantial evidence (e.g., Meadows et al. 1995) that some, and probably most, of each condensation's mass disintegrated high in the Jovian atmosphere, large enough residual masses of 11 of the components penetrated into the lower stratosphere, or perhaps still deeper, where they exploded and generated huge ejecta clouds, some of which were imaged with the HST (Hammel et al. 1995). This was hardly what one would expect of a strengthless assemblage of hundred-meter-sized cometesimals!

Also of concern is the sensitivity of the strengthless agglomerate models to the comet's rotation. Both Asphaug \& Benz and Solem found that retrograde progenitor rotation states yield train configurations containing a dominant central clump, instead of the observed quasi-uniform strand of clumps. The validity of their conclusion that the parent comet could not have been a retrograde rotator is limited by their model's assumptions and does not apply as a general rotation constraint.

The sensitive dependence of the strengthless agglomerate models on the choice of bulk density is even more worrisome. While Shoemaker-Levy 9 yields a plausible value for the bulk density, the application to other tidally split comets leads to far less satisfactory results. For $\mathrm{P} /$ Brooks 2 , which could not have split at a jovicentric distance of less than two planet's radii (cf. Sec. 3.5), these models would unquestionably predict a significantly lower density. Worse yet, the strengthless assemblage paradigm offers particularly discouraging results for the sungrazing group of comets. Two of them, 1882 II and IkeyaSeki 1965 VIII (both mentioned in Sec. 1), had a nearly identical perihelion distance of 1.67 solar radii, but $1882 \mathrm{II}$, the much brighter (and almost certainly much larger) of the two was observed after perihelion to have split into six discrete condensations (Kreutz 1888), while 1965 VIII into only two (Sekanina 1977). Furthermore, only one possible secondary condensation was reported (Roemer 1965) for another well-observed member of the sungrazing group, Pereyra (1963 V), and none for $1843 \mathrm{I}$ (=1843 D1) and $1880 \mathrm{I}$ $(=1880 \mathrm{C} 1)$, even though their perihelia were still closer to the Sun, between 1.09 and 1.19 solar radii. Since all the sungrazers have a single common parent (Marsden 1967, 1989), major variations in their effective bulk density are unlikely and the examples provide strong evidence against the validity of the strengthless agglomerate models.

One can point out additional problems with the strengthless models: doubts whether they can explain the observed nuclear train orientation more than eight months after the breakup (Sec. 4.2), an issue not addressed by the Asphaug-Benz-Solem papers; a contra- 
diction in the tacit assumption that while the dynamical behavior of the assemblage is governed purely by self-gravity, the cometesimals themselves are structurally so cohesive that their strength is not even questioned; and a highly problematic long-term dynamical stability of strengthless agglomerates, a major problem for all sand bank models. Aggregate structures dominated by water ice were recently shown to possess a tensile strength on the order of $10^{-3}$ bar (Greenberg et al. 1995). All these arguments support the notion expressed by Whipple a long time ago (Sec. 5.1) that gravitational coherence alone cannot provide the basis for a realistic model of cometary nuclei.

\subsection{Models for a discrete mass of limited mechanical strength}

Tidal splitting of a discrete nucleus that possesses some, however limited, strength is governed by different conditions. As mentioned in Secs. 5.1 and 5.2, cohesion in such nuclei of aggregate structure should vary due to unevenly strong mechanical bonds among its building blocks or due to uneven cementing of the interiors of the individual blocks, or both. Considering a self-gravitating, incompressible elastic sphere, Aggarwal \& Oberbeck (1974) showed that fracture starts either at the body's center or on its surface. In general, their result can be written in a form analogous to (5.1),

$$
\frac{\rho}{\rho_{0}}\left(\frac{\Delta}{R_{0}}\right)^{3}<k \frac{P_{\mathrm{c}}}{U},
$$

where $k$ is a constant on the order of unity. When fracture starts at the surface, $U$ equals $T$, the body's tensile strength. When it starts at the center, $U=T+P_{\mathrm{c}}$. Aggarwal \& Oberbeck argued that fracture is completed when the tensile strength is exceeded by the greatest principal stress both at the center and on the surface of the body. Dobrovolskis (1990) pointed out, however, that Aggarwal \& Oberbeck's approach underestimated the extent of fracture because their calculation of the stress field did not account for its changes as the fissure propagates. In any case, the critical tensile strength at which the body would begin to break apart varies as the central pressure and is therefore proportional to the square of the body's size. In scenarios with fixed values of $\rho, \rho_{0}$, $R_{0}$, and $\Delta$, the larger a comet's nucleus is the easier it is to split it tidally. The size dependence represents a fundamental difference between the behavior of strengthless agglomerates and nuclei that are at least weakly cemented. It is noted that the proposed size dependence is consistent with the uneven numbers of condensations observed in the sungrazers 1882 II and 1965 VIII (Sec. 5.2).

Secondary fragmentation is one of the observed phenomena that makes the concept of discrete cometary nuclei of limited and variable strength very attractive. The fact that the products of a secondary fragmentation event appeared as two distinct condensations, rather than an elongated cloud of dust, testifies to the presence of a dominant mass in each daughter condensation. Their diffuse appearance suggests that much, and possibly all, of the involved dust population was formed during the discrete event. Otherwise one would have to postulate a bimodal velocity distribution in the parent condensation's dust cloud, a premise with no physical or dynamical rationale and difficult to defend.

Events of secondary fragmentation can readily be understood in the framework of a discrete nucleus as a result of a gradual fissure propagation. Asphaug \& Benz $(1994,1996)$ argued that a body of any realistic density could not have been broken up into $\sim 21$ pieces by the tidal forces, regardless of its strength. They proposed that if the comet was not a strengthless "rubble pile" to begin with, it would have to be a structurally weak aggregate shattered by impact during its inbound passage through the Jovian ring plane. This scenario fails to explain the breakup of $\mathrm{P} /$ Brooks 2, which never approached the planet close enough to cross the ring. Since the initial number of major fragments of comet 
Shoemaker-Levy 9 was just 10 to 12 (Sec. 4.3), Asphaug \& Benz's objection becomes irrelevant. Also, their argument applies neither to scenarios involving additional forces, such as rotational stresses, that can assist the tides in breaking the nucleus up, nor to irregular bodies and/or to bodies of nonuniform strength. In fact, Greenberg et al. (1995) show that sequential tidal splitting of the parent nucleus of a nonzero strength can result in still more than $\sim 20$ pieces, depending on the comet's original nuclear size.

However, Asphaug \& Benz's suggestion that growing cracks in a nucleus of some intrinsic strength may stall is of interest, paralleling somewhat our argument in Paper 1. The implications are that a body of uniform strength is pure fiction and any arguments against its expected behavior are meaningless. A crack could stall whenever it encounters a mass of greater strength during its propagation through the nucleus. As the comet rotates, the centrifugal force would assist the tides in some parts of the nucleus, depending on the orientation of the spin vector. Even for a nonrotating comet, the configuration of regions of variable strength in the nucleus interior should be changing rapidly relative to Jupiter (and its tidal field) near perijove because of the sharply curved orbit. An obvious inference is that there should have been large fragments that had survived the Jovian encounter cracked but not completely broken and that some of the cracks would have been extended to the point of fracture at later times in those among the fragments that happened to have been spun up as a result of the collisional angular momentum redistribution in the cloud of debris.

The plausibility of the concept of discrete nuclei of limited and variable strength is also illustrated by other idiosyncrasies of the condensations of Shoemaker-Levy 9, both in interplanetary space and upon entering the Jovian atmosphere. One of these peculiarities is the gradual disappearance of a condensation, which is particularly well documented by the HST observations of P1 (Fig. 2 of Weaver et al. 1995), but was obviously also experienced by $\mathrm{J}, \mathrm{M}$, and $\mathrm{P} 2 \mathrm{~b}$. Common to these condensations was apparently their extremely poor cementing throughout their interiors on such scales that no fragment of a size detectable by the HST could survive even in interplanetary space. This critical size at the comet's distance is about $1 \mathrm{~km}$ in diameter, so the gradual diappearance of these condensations does not provide a very strict limit on the maximum fragment size and on the density of lines of extreme structural weakness in these objects.

The next group includes other off-train condensations, such as B, F, etc., which did not disintegrate during the months before impact but generated no detectable ejecta. Since stresses (such as rotational) acting on comets and their fragments in interplanetary space are generally lower than the tidal forces very close to Jupiter, it appears that lines of high structural weakness were less densely distributed in the interiors of these fragments, but that areas of some structural weakness were still sufficiently extensive in these objects for their tidal breakup shortly before entering the atmosphere (Sekanina 1993).

Finally, the on-train condensations apparently contained kilometer-sized fragments in which areas of high structural weakness were still less common, so that these fragments survived the tidal action and began to fragment precipitously only under the effects of aerodynamic pressure, which for the impact velocity of Shoemaker-Levy 9 began to exceed the tidal force at altitudes of about $300 \mathrm{~km}$ or so above the 1-bar level.

These groups of fragments clearly correlate with the classes introduced by Hammel et al. (1995) and suggest that the distribution of lines of extreme structural weakness is this classification's criterion. However, one should not think in terms of discrete categories; instead, each fragment is likely to have its own position in the hierarchy of structural strength. One observational implication is that the off-train condensations of structurally weaker fragments should have generally appeared brighter than the ontrain condensations, because a greater mass fraction of the off-train condensations was 
contained in the debris near the lower end of the size spectrum, so they had a higher apparent cross-sectional area per unit mass. This effect was particularly well illustrated by the first two condensations to crash, A and B, as A was in fact fainter, yet apparently much more massive. Of the on-train condensations, one example of an excessively fragile fragment was $Q$, which over a time gave birth to perhaps as many as five secondary fragments. This condensation had long been the brightest component of the train, yet the impact of Q1 was a relatively disappointing event.

The high susceptibility of cometary objects to fragmentation during flight through the Earth's atmosphere has long been known from observational evidence accumulated in the field of meteor physics (e.g., McCrosky \& Ceplecha 1970). In fact, fragmentationespecially discrete fragmentation events, which trigger flares or outbursts along the atmospheric path-is clearly the dominant ablation process for massive cometary impactors. Borovička \& Spurný (1995) recently analyzed a photographed cometary bolide, which morphologically represents a good analogue for the fragments of comet ShoemakerLevy 9. The bolide's maximum brightness normalized to a distance of $100 \mathrm{~km}$ reached panchromatic magnitude -21.5 , its bulk density was $\sim 0.1 \mathrm{~g} \mathrm{~cm}^{3}$, and its initial (preatmospheric) mass $\sim 5$ tons. Borovička \& Spurný also found that along the entire luminous path the bolide's flight was only marginally decelerated by the atmosphere. The bolide became visible at an altitude of $99 \mathrm{~km}$ above sea level, where the dynamic pressure reached $\sim 2$ mbar and the atmospheric pressure was only $0.4 \mu \mathrm{bar}$. An equivalent altitude in the Jovian atmosphere is $\sim 380 \mathrm{~km}$ above 1 bar. The bolide disintegrated entirely by the time it reached an altitude of $59 \mathrm{~km}$ (an equivalent Jovian altitude of $\sim 190 \mathrm{~km}$ above $1 \mathrm{bar}$ ), at a dynamic pressure of $\sim 1$ bar and an atmospheric pressure of 0.25 mbar. Borovička \& Spurný's modelling of the object's light curve shows that almost 50 percent of the initial mass was lost in the brightest flare alone, whose FWHM duration was $\sim 0.05$ second, and that the residual mass after this event amounted to less than 1 percent of the initial mass. This outburst was observed at an altitude of $67 \mathrm{~km}$, where the dynamic pressure reached $\sim 0.4$ bar and the atmospheric pressure was 0.08 mbar. The fragments of Shoemaker-Levy 9 were subjected to the same dynamic pressure at an altitude of $\sim 200 \mathrm{~km}$ above 1 bar, where the atmospheric pressure was $\sim 0.15$ mbar.

To summarize, the paradigm of a cometary nucleus that possesses a limited but variable strength avoids conceptual pitfalls of the strengthless agglomerate models. The results for the tidal breakup are no longer critically sensitive to the bulk density, for which values significantly lower than $0.5 \mathrm{~g} \mathrm{~cm}^{3}$ are preferred. This limit is based not on the results obtained from the strengthless agglomerate models, but from the simple application of Eq. (5.1) to P/Brooks 2. The Shoemaker-Levy 9 progenitor could have begun to break apart perhaps as early as one hour or more before perijove, especially if its nuclear dimensions were relatively large (Sec. 5.4). The interpretation of secondary fragmentation and the explanation of the dramatic differences in the behavior among the condensations both before and upon their atmospheric entry are thus logical outgrowths of the fundamental conclusion on low and highly variable strength of tidally generated fragments and their products.

\subsection{Sizes of the progenitor nucleus and its fragments}

The dimensions of the progenitor nucleus and its major fragments have been a subject of continuing controversy. A relatively soft upper limit on the progenitor's photometric cross section results from a failure to find the comet on prediscovery exposures taken with the 100-cm Schmidt telescope at the European Southern Observatory in March 1992 (Tancredi et al. 1993, Tancredi \& Lindgren 1994), on which the limiting magnitude for an object having the comet's motion was $B=21.3$. Even the largest estimates for the 
nuclear size indicate that the comet, if inactive, would have been at least 0.5 magnitude fainter, assuming appropriate values for the albedo and the phase coefficient.

In the papers by Scotti \& Melosh (1993), by Asphaug \& Benz (1994, 1996), by Solem (1994, 1995), and by Chernetenko \& Medvedev (1994) the effective diameter of the original nucleus was estimated at $\lesssim 2 \mathrm{~km}$. Scotti \& Melosh found $2.3 \mathrm{~km}$, but they used an early orbit whose 1992 perijove distance was too large. Refined orbits yielded a smaller miss distance (Yeomans \& Chodas 1994), requiring a revision of Scotti \& Melosh's value to $1.8 \mathrm{~km}$. Asphaug \& Benz, employing a recent orbit, derived an effective diameter of $1.5 \mathrm{~km}$, while Solem obtained $1.8 \mathrm{~km}$, and Chernetenko \& Medvedev, $1.1 \mathrm{~km}$.

The agreement among these results is not surprising, because they all were determined from the same observed quantity - the length of the nuclear train, always interpreted as a product of radial differential perturbations by Jupiter. Only in two of the four studies was the issue of the trails addressed at all, very briefly in either case. Asphaug \& Benz's discussion was limited to an obvious remark that trails of debris could be expected on either side of the major clumps, but they offered no quantitative information. Scotti \& Melosh concluded that the trails were either made up of remnants of a dust coma that the comet had possessed before its close encounter with Jupiter, or consisted of dust liberated during the breakup and subsequently perturbed by various forces. The extent of the trails was noted by these authors to correspond to a diameter of $\sim 20-35$ times the diameter of the parent comet but they did not elaborate on the significance of this finding. The other two papers ignored the existence of the trails altogether and none of the four studies paid any attention to the system of tails or to the sector of material to the north of the train and the trails.

In the meantime, the comet's first observations by the HST, made on July 1, 1993, were analyzed by Weaver et al. (1994). After subtracting the light of the surrounding comae, the magnitudes of the central nuclei in the 11 brightest condensations were calculated to imply effective diameters in the range from 2.5 to $4.3 \mathrm{~km}$, at an assumed geometric albedo of 0.04. Even though Weaver et al. remarked that the derived nuclear magnitudes may not have been entirely free from a contamination by residual dust in the employed $3 \times 3$ pixel box centered on the brightest pixel, the effect could not possibly have amounted to $>3$ magnitudes, nor could the albedo have been underestimated by a factor of $>20$ to make the results compatible with the progenitor's diameter of $\lesssim 2 \mathrm{~km}$.

Weaver et al. (1995) subsequently applied the same technique to the HST observations from January and March 1994, finding that the spatial brightness distribution in the condensations could not be fitted by a simple model and that there was no reliable way of deconvolving the contributions by any unresolved sources from the surrounding dust clouds. On the other hand, Sekanina's (1995a,b) application of his independent deconvolution technique to the HST images taken in January, March, and early July 1994 resulted in positive detections of unresolved sources in nearly all condensations under investigation. The dimensions calculated for the major fragments were virtually independent of the law employed to approximate the brightness distribution of the extended source (the surrounding dust cloud) and agreed closely with those derived by Weaver et al. (1994) from the July 1993 data.

Other, independent lines of evidence also suggest that the original nucleus could not possibly be $\lesssim 2 \mathrm{~km}$ in diameter. Using the formalism of sequential tidal fragmentation introduced by Dobrovolskis (1990), Greenberg et al. (1995) showed that with a derived tensile strength of 0.0027 bar and a plausible bulk density of $\sim 0.3 \mathrm{~g} \mathrm{~cm}^{3}$, the nucleus of Shoemaker-Levy 9 could not break up at all unless its diameter was at least $\sim 5 \mathrm{~km}$. In addition, their numbers imply that the nucleus could not break up into 10-12 pieces (Sec. 4.3) unless its diameter was at least $7-8 \mathrm{~km}$. Greenberg et al. noted an encouraging 
agreement between their results and the nuclear sizes inferred by Weaver et al. (1994) and by Sekanina et al. (1994). For a bulk density of $0.2 \mathrm{~g} \mathrm{~cm}^{3}$, assumed in this paper, the comet's nucleus could not split unless it was at least $6 \mathrm{~km}$ in diameter and could not split into the observed number of major fragments unless at least $9-10 \mathrm{~km}$ in diameter. Also of interest are the implications for $1882 \mathrm{II}, 1965 \mathrm{VIII}$, and the parent comet of $\mathrm{P} /$ Brooks 2, whose minimum diameters are found to be equal, respectively, to $12 \mathrm{~km}$, $8 \mathrm{~km}$, and $11 \mathrm{~km}$. On the other hand, if $1963 \mathrm{~V}$ did not break up, its nucleus could not be more than $4 \mathrm{~km}$ across.

Further evidence that the parent nucleus of Shoemaker-Levy 9 was $\gg 2 \mathrm{~km}$ in diameter is provided by the studies of various aspects of the impact phenomena.

From their analysis of the optical depth distribution of the dark impact debris on Jupiter imaged with the HST between 1 day and 1 month after the last impact, R. A. West et al. (1995) concluded that the mean particle radius was between 0.2 and $0.3 \mu \mathrm{m}$ and their total volume was equal to that of a sphere $1.0 \mathrm{~km}$ in diameter. Since these aerosol particles are believed to have represented condensates of supersaturated vapor originating from the hot gas in the raising plumes of debris, they consisted primarily - and perhaps exclusively - of the impactor's mass and, unlike in the original cometary environment, had densities close to the mineralogical densities of the involved materials. R. A. West et al. adopted a density of $2 \mathrm{~g} \mathrm{~cm}^{3}$, which yields a total of $1.0 \times 10^{15} \mathrm{~g}$ for this optically recovered mass of the refractory material, which alone corresponds to an effective diameter of $2.1 \mathrm{~km}$ for the comet's plausible bulk density of $0.2 \mathrm{~g} \mathrm{~cm}^{3}$ (cf. Secs. 5.2 and 5.3). There is no doubt whatsoever that this optically recovered aerosol mass represents only a fraction of the total recondensed refractory mass of the fragments, which, in turn, represents only a small fraction of the total mass delivered to Jupiter by the fragments and by the dust clouds in which the fragments were immersed before impact. Even this total delivered mass obviously does not represent the entire mass of the original comet, although the two may be comparable in magnitude.

The incompleteness of the optically recovered aerosol mass is plainly illustrated by the fact that the contributions to the dark debris from most off-train condensations remained undetected. Besides, R. A. West et al. (1995) emphasize that significant nucleation requires supersaturation and that cooling below the saturation point is not a sufficient condition for the aerosol formation. They also find that, in a dense plume of debris, silicates and similar refractory materials could condense into grains larger than $10 \mu \mathrm{m}$ in radius, whose sedimentation times in the Jovian atmosphere are only a fraction of 1 day. Such large particles obviously could not survive in a debris imaged days or weeks after impact. When R. A. West et al.'s results are combined with Vanýsek's (1995) estimate that only $\sim 1$ percent of the delivered mass should have contributed to the recondensation process, one finds a total delivered cometary mass of $\sim 10^{17} \mathrm{~g}$. And since the optically recovered aerosol mass was probably derived from the recondensed residual mass involved in the explosions in the lower stratosphere and/or the troposphere, it would not include the fraction of the original mass of the impactors that was lost by their precipitous fragmentation in the upper atmosphere prior to the explosions and may have been responsible for, or contributed to, the detected heating of the stratosphere (e.g., Lellouch et al. 1995, Bézard et al. 1995). This missing mass has remained unaccounted for, even though it may have represented a significant fraction of the initial mass of the large fragments. Finally, from observations at millimeter wavelengths, additional substantial amounts of the delivered mass were identified by Lellouch et al. (1995) in the form of volatile compounds, concentrated near the $0.5 \mathrm{mbar}$ pressure level and probably involved in the shock chemistry. For the fragment $\mathrm{K}$, for example, a mass of $2.5 \times 10^{14} \mathrm{~g}$ of carbon monoxide was detected several hours after impact (Lellouch, this volume), which alone 
represents $\sim 30$ percent of the total mass of the Asphaug-Benz parent comet. Recovery of all these impressively large amounts of mass from an original nucleus of less than $2 \mathrm{~km}$ in diameter would surely represent a humpty-dumpty feat of unrivalled proportions.

\subsection{Rotation model for the progenitor nucleus and collisional evolution of the debris}

One of the first results of the numerical experiments conducted in Paper 1 was a finding that equivalent values could be established for an initial radial separation of the fragments or for their orbital velocity increment or for various combinations of these quantities so that they yielded identical temporal variations in the nuclear train's apparent evolution (its length and orientation), a fact that can also be derived from the virial theorem. If the breakup is assumed to have occurred at closest approach, the relevant values are $1.26 \mathrm{~km}$ for the radial separation (that is, the nucleus diameter) and $0.17 \mathrm{~m} \mathrm{~s}^{-1}$ for the orbital velocity increment. Thus, the breakup of the progenitor $1.26 \mathrm{~km}$ in diameter represents only one of an infinite number of possible solutions, based on a number of assumptions regarding the time of the event (exactly at perijove) and the comet's rotation vector (no rotation or the axis aligned with the orbital velocity vector). This is such an exceptionally special case that the probability of its having actually taken place is virtually nil. The equivalence of effects due to Jovian perturbations and due to an orbital velocity impulse signifies a basic dynamical indeterminacy of the problem, which is also reflected in the major role of the comet's rotation recognized by Asphaug \& Benz $(1994,1996)$ and by Solem $(1994,1995)$ and which makes the tight constraints on the parent comet's size and bulk density in these models vulnerable and suspect.

A Monte Carlo simulation of ubiquitous low-velocity particle-particle collisions, carried out in Paper 1, showed that the initial rotational velocities were rearranged into a rapidly "thermalized" distribution, characterized by a long tail of fairly high velocities (up to $\sim 7 \mathrm{~m} \mathrm{~s}^{-1}$ ) for the debris that eventually populated far regions of the trails. The period of intense particle-particle collisions was estimated to have continued for at least a few hours, at which time the systematic forces began to dominate. The particle mass distribution of the fragments appears to have been relatively flat near the upper end of the size spectrum but steeper for pebble-sized and smaller debris. Fine dust effectively provided a temporary viscous medium for the major fragments. Dimensions of fragments populating the west-southwestern trail probably ranged from several hundred meters down to a few centimeters, the latter constraint being dictated by the absence of measurable solar radiation pressure effects. The debris in the east-northeastern trail was mostly submillimeter- and millimeter-sized. All the debris to the north of the train-trail boundary was affected by solar radiation pressure and made up of particles that were microns to several millimeters across, the size being the largest near the boundary.

To constrain the comet's bulk properties, a rotational model was formulated by us in Paper 1 and a search was initiated for solutions consistent with evidence on the nuclear train and the trails, while also accommodating limited information on the tails and the sector of material. The maximum dynamically plausible train and trail lengths, searched for as functions of a location on the nucleus, depend on the nuclear dimensions and the rotation vector of the parent comet, on the effective breakup time (Sec. 4.2), on the particle-mass distribution of the debris, and on the collisional-velocity enhancement factors. Although no unique solution could be derived, models for the parent comet that fitted the constraints best implied a nuclear diameter of $\sim 10 \mathrm{~km}$, a spin axis nearly in the jovicentric orbital plane, and a short rotation period, perhaps 7-8 hours. For a bulk density of $0.2 \mathrm{~g} \mathrm{~cm}^{3}$, the net tidal stress is calculated to have been 0.0038 bar at perijove, 0.0008 bar 1 hour earlier, and 0.0002 bar 2 hours earlier, comparable with the central 
gravitational pressure and the centrifugal stress due to rotation. It thus appears that the comet's spin assisted the tidal forces in splitting the nucleus apart.

\section{Summary and conclusions}

The events experienced by comet Shoemaker-Levy 9 near Jupiter in early July 1992 began with fissures propagating throughout its nucleus, about $10 \mathrm{~km}$ in diameter or $10^{17} \mathrm{~g}$ in mass at an assumed density of $0.2 \mathrm{~g} \mathrm{~cm}^{3}$. The cracks were caused by tidal stresses exerted by the planet, with some assistance from the comet's rotation. Probably even before reaching perijove, the inflicted structural failures resulted in the body's gradual breakup, first into a couple of large fragments accompanied by immense amounts of smallsized debris. Because of a distribution of rotation velocities, collisions became inevitable and, together with the continuing tidal forces, contributed to further fragmentation. The collisional velocity distribution rapidly "thermalized" and developed a long tail, populated by particulates with relative velocities of up to $\sim 7 \mathrm{~m} \mathrm{~s}^{-1}$. Intensive collisions did not terminate until after perijove, defining the effective time of breakup (dynamical separation). The 10-12 largest fragments contained apparently close to 90 percent of the total mass of the progenitor. The largest fragment was estimated to have been at least $4 \mathrm{~km}$ in diameter. A mean fragment size gradually decreased from the train to the two trails, the tails, and the sector of material that contained microscopic debris. Definite evidence for discrete events of secondary fragmentation indicates that the comet's disintegration continued long after its 1992 encounter with Jupiter. Observed effects on the unevenly susceptible fragments provide intriguing information on the complex morphology of the comet's nucleus interior. The orbital calculations offer an independent insight by showing that fragments that ended up nearer the planet at the end of the collisional period remained so throughout the orbit until collision, while fragments with greater velocity increments in the direction of the comet's motion had larger orbital dimensions and impacted Jupiter later.

The comet's fragments of the estimated mass will have delivered a total energy of tens of millions of megatons of TNT upon impact. Much of this energy was rapidly dissipated over huge volumes of the Jovian atmosphere in the early phase of each fragment's entry and only a fraction was apparently transformed into more persisting, readily detectable effects. Whereas the impact phenomena provide critical information on the nature of the fragments' interaction with the Jovian atmosphere, the comet's tidal disruption deserves attention in a broader context, including the role of nuclear splitting in the evolution of comets. A particularly diagnostic property concerns systematic differences in fragment configurations of tidally and nontidally split objects. Whatever the mechanism(s) of nontidal breakup may be (Sec. 1), it is well known (Sekanina 1977, 1982) that the configuration of fragments is, in these cases, controlled primarily by differential forces acting along the direction of the radius vector. Relative to the principal (parent) nucleus, which is usually (but not necessarily) the brightest component, the companions are lined up approximately along the antisolar direction shortly after their separation, but rotate their positions gradually with time and end up eventually-if they are still observable-in the direction of the reverse orbital-velocity vector, that is, they follow the parent object in its heliocentric orbit. Hence, the characteristic attribute of such configurations is that the parent nucleus is always situated at the leading end of the fragment lineup. This dynamical evolution is of course readily predictable from considerations of the orbital angular momentum and is indeed consistent with observations of fragments of most split comets. For only three among the split comets with more than two components ever observed was the brightest condensation situated at a "wrong" location, after 
each of them had broken tidally in the immediate proximity of the Sun or Jupiter: the sungrazer 1882 II, $\mathrm{P} /$ Brooks 2, and Shoemaker-Levy 9. This evidence suggests that the fragment configurations of tidally split comets are determined primarily by the conditions at breakup and not by the differential forces that the fragments are subjected to following their separation.

I thank H. A. Weaver for providing his measurements of the offsets of the component Q2 from Q1 on the HST images. I also thank J.V. Scotti for communicating his and T.S. Metcalfe's results on the dust trails and tails before publication. This research was carried out by the Jet Propulsion Laboratory, California Institute of Technology, under contract with the National Aeronautics and Space Administration and was supported in part through Grants GO-5021 and GO-5624 from the Space Telescope Science Institute, operated by the Association of Universities for Research in Astronomy, Inc., under contract with the National Aeronautics and Space Administration.

\section{REFERENCES}

Aggarwal, H. R. \& Oberbeck, V. R. 1974 Roche limit of a solid body. Astrophys. J. 191, $577-588$.

Asphaug, E. \& Benz, W. 1994 Density of comet Shoemaker-Levy 9 deduced by modelling breakup of the parent "rubble pile." Nature 370, 120-124.

Asphaug, E. \& Benz, W. 1996 The tidal disruption of strengthless bodies: lessons from comet Shoemaker-Levy 9. Icarus, in press.

BARNARD, E. E. 1889 Discovery and observations of companions to comet $1889 \ldots$ (Brooks July 6). Astron. Nachr. 122, 267-268.

BARNARD, E. E. 1890 Physical and micrometrical observations of the companions to comet $1889 \mathrm{~V}$ (Brooks). Astron. Nachr. 125, 177-196.

Bézard, B., Griffith, C. A., Kelly, D., Lacy, J., Greathouse, T. \& Orton, G. 1995 MidIR high-resolution spectroscopy of the SL9 impact sites: Temperature and HCN retrievals. Poster paper presented at IAU Colloq. No. 156 The Collision of Comet P/Shoemaker-Levy 9 and Jupiter, Baltimore, Maryland, May 1995.

BoroviČKA, J. \& SPURNÝ, P. 1995 Radiation study of two very bright terrestrial bolides. Poster paper presented at IAU Colloq. No. 156 The Collision of Comet P/ShoemakerLevy 9 and Jupiter. ST ScI.

Boss, A. P. 1994 Tidal disruption of periodic comet Shoemaker-Levy 9 and a constraint on its mean density. Icarus 107, 422-426.

Chernetenko, Y. A. \& Medvedev, Y. D. 1994 Estimate of the Shoemaker-Levy 9 nucleus size from position observations. Planet. Space Sci. 42, 95-96.

Chodas, P. W. \& Yeomans, D. K. 1994 Comet Shoemaker-Levy 9 impact times and impact geometries. Bull. Amer. Astron. Soc. 26, 1569.

Dobrovolskis, A. R. 1990 Tidal distuption of solid bodies. Icarus 88, 24-38.

Feldman, P. D., Weaver, H. A., Boice, D. C. \& Stern, S. A. 1996 HST observations of $\mathrm{Mg}^{+}$in outburst from comet $\mathrm{D} /$ Shoemaker-Levy 9 . Icarus, in press.

Greenberg, J. M., Mizutani, H. \& Yamamoto, T. 1995 A new derivation of the tensile strength of cometary nuclei: application to comet Shoemaker-Levy 9. Astron. Astrophys. 295, L35-L38.

Hammel, H. B., Beebe, R. F., Ingersoll, A. P., Orton, G. S., Mills, J. R., Simon, A. A., Chodas, P., Clarke, J. T., De Jong, E., Dowling, T. E., Harrington, J., Huber, L. F., Karkoschka, E., Santori, C. M., Tolgo, A., Yeomans, D. \& West, R. A. 1995 HST imaging of atmospheric phenomena created by the impact of comet ShoemakerLevy 9. Science 267, 1288-1296. 
JewitT, D., LuU, J. \& ChEN, J. 1993 Physical properties of split comet Shoemaker-Levy 9. Bull. Amer. Astron. Soc. 25, 1042.

Jewitt, D. \& Trentham, N. 1994 Periodic comet Shoemaker-Levy 9 (1993e). IAU Circ. No. 5999.

Kreutz, H. 1888 Untersuchungen über des Cometensystem 1843 I, 1880 I und 1882 II. Publ. Sternw. Kiel No. 3, pp. 1-111.

LehKÝ, M. 1994 Periodic comet Shoemaker-Levy 9 (1993e). Int. Comet Q. 16, 35.

Lellouch, E., Paubert, G., Moreno, R., Festou, M. C., Bézard, B., BockeléeMorvan, D., Colom, P., Crovisier, J., Encrenaz, T., Gautier, D., Marten, A., Despois, D., Strobel, D. F. \& Sievers, A. 1995 Chemical and thermal response of Jupiter's atmosphere following the impact of comet Shoemaker-Levy 9. Nature 373, 592595.

Lyttleton, R. A. 1953 The Comets and Their Origin. Cambridge University.

MARSDEN, B. G. 1967 The sungrazing comet group. Astron. J. 72, 1170-1183.

MARsden, B. G. 1989 The sungrazing comet group. II. Astron. J. 98, 2306-2321.

MCCrosky, R. E. \& CePlecha, Z. 1970 Fireballs and physical theory of meteors. Bull. Astron. Inst. Czech. 21, 271-296.

Meadows, V., Crisp, D., Orton, G., Brooke, T. \& Spencer, J. 1995 AAT IRIS observations of the SL-9 impacts and initial fireball evolution. In European ShoemakerLevy 9/Jupiter Workshop (eds. R. West \& H. Böhnhardt), pp. 129-134. European Southern Observatory.

Mumma, M. J. 1995 Personal communication.

Noll, K. S. \& SMITh, T. E. 1994 Periodic comet Shoemaker-Levy 9 (1993e). IAU Circ. No. 6010.

Renz, F. 1889 Ueber die Begleiter des Cometen 1889...(Brooks Juli 6). Astron. Nachr. 122, 413-416.

Rettig, T. W., Mumma, M. J., Tegler, S. C. \& Hahn, J. 1994 Are the fragments of comet Shoemaker-Levy 9 swarms of meter-sized planetesimals? Bull. Amer. Astron. Soc. 26, 862.

RoEMER, E. 1965 Observations of comets and minor planets. Astron. J. 70, 397-402.

Schatzman, E. 1953 La structure et l'evolution des noyaux cometaires. Mém. $8^{\circ}$ Soc. Roy. Sci. Liège (Sér. 4) 13 (Fasc. 1-2), 313-323.

ScotTI, J. V. 1993 Periodic comet Shoemaker-Levy 9. Minor Planet Circ. Nos. 21988-21989.

ScotTr, J. V. \& Melosh, H. J. 1993 Estimate of the size of comet Shoemaker-Levy 9 from a tidal breakup model. Nature 365, 733-735.

Scotti, J. V. \& Metcalfe, T. S. 1995 Personal communication.

SEKANINA, Z. 1977 Relative motions of fragments of the split comets. I. A new approach. Icarus 30, 574-594.

Sekanina, Z. 1982 The problem of split comets in review. In Comets (ed. L. L. Wilkening), pp. 251-287. University of Arizona, Tucson.

SEKANINA, Z. 1993 Disintegration phenomena expected during collision of comet ShoemakerLevy 9 with Jupiter. Science 262, 382-387.

Sekanina, Z. 1995a Nuclei of comet Shoemaker-Levy 9 on images taken with the Hubble Space Telescope. In European Shoemaker-Levy 9/Jupiter Workshop (ed. R. West \& H. Böhnhardt), pp. 29-35. European Southern Observatory.

SEKANINA, Z. 1995b Evidence on sizes and fragmentation of the nuclei of comet ShoemakerLevy 9 from Hubble Space Telescope images. Astron. Astrophys. 304, 296-316.

Sekanina, Z., Chodas, P. W. \& Yeomans, D. K. 1994 Tidal disruption and the appearance of periodic comet Shoemaker-Levy 9. Astron. Astrophys. 289, 607-636. (Paper 1.)

Sekanina, Z., Chodas, P. W. \& Yeomans, D. K. 1995 In preparation.

SEKanina, Z. \& Yeomans, D. K. 1985 Orbital motion, nucleus precession, and splitting of periodic comet Brooks 2. Astron. J. 90, 2335-2352. 
Solem, J. C. 1994 Density and size of comet Shoemaker-Levy 9 deduced from a tidal breakup model. Nature 370, 349-351.

SolEM, J. C. 1995 Cometary breakup calculations based on a gravitationally-bound agglomeration model: the density and size of Shoemaker-Levy 9. Astron. Astrophys. 302, 596-608.

TANCREDI, G. \& LindgREN, M. 1994 Searching for comets encountering Jupiter: First campaign. Icarus 107, 311-321.

Tancredi, G., Lindgren, M. \& Lagerkvist, C.-1. 1993 Periodic comet Shoemaker-Levy 9 (1993e). IAU Circ. No. 5892.

VANÝSEK, V. 1995 A note on the fragment size of SL-9 and debris field. In European ShoemakerLevy 9/Jupiter Workshop (ed. R. West \& H. Böhnhardt), pp. 297-298. European Southern Observatory.

Vorontsov-Velyaminov, B. 1946 Structure and mass of cometary nuclei. Astrophys. J. 104, 226-233.

Weaver, H. A. 1994 Periodic comet Shoemaker-Levy 9 (1993e). IAU Circ. Nos. 5947 \& 5973.

WeaVer, H. A. 1995 Personal communication.

Weaver, H. A., Feldman, P. D., A'Hearn, M. F., Arpigny, C., Brown, R. A., Helin, E. F., Levy, D. H., Marsden, B. G., Meech, K. J., Larson, S. M., Noll, K. S., Scotti, J. V., Sekanina, Z., Shoemaker, C. S., Shoemaker, E. M., Smith, T. E., Storrs, A. D., Yeomans, D. K. \& Zellner, B. 1994a Hubble Space Telescope observations of comet $P$ /Shoemaker-Levy 9 (1993e). Science 263, 787-791.

Weaver, H. A., A'Hearn, M. F., Arpigny, C., Boice, D. C., Feldman, P. D., Larson, S. M., Lamy, P., Levy, D. H., Marsden, B. G., Meech, K. J., Noll, K. S., ScotTi, J. V., Sekanina, Z., Shoemaker, C. S., Shoemaker, E. M., Smith, T. E., Stern, S. A., Storrs, A. D., Trauger, J. T., Yeomans, D. K. \& Zellner, B. 1995 The Hubble Space Telescope (HST) observing campaign on comet Shoemaker-Levy 9. Science 267, 1282-1288.

WeIss, E. 1889 Ueber die Erscheinungen am Cometen 1889... (Brooks Juli 6). Astron. Nachr. 122, 313-316.

Weissman, P. 1994 The big fizzle is coming. Nature 370, 94-95.

West, R. A., Karkoschka, E., Friedson, A. J., Seymour, M., Baines, K. H. \& Hammel, H. B. 1995 Impact debris particles in Jupiter's stratosphere. Science 267, 1296-1301.

WEST, R. M., HooK, R. N. \& HAINAUT, O. 1995 A morphological study of SL-9 CCD images obtained at La Silla (July 1-15, 1994). In European Shoemaker-Levy 9/Jupiter Workshop (ed. R. West \& H. Böhnhardt), pp. 5-10. European Southern Observatory.

Whipple, F. L. 1950 A comet model. I. The acceleration of comet Encke. Astrophys. J. 111, $375-394$.

Whipple, F. L. 1951 A comet model. II. Physical relations for comets and meteors. Astrophys. J. $113,464-474$.

Whipple, F. L. 1961 Problems of the cometary nucleus. Astron. J. 66, 375-380.

WhIPple, F. L. 1963 On the structure of the cometary nucleus. In The Moon, Meteorites, and Comets (ed. B. M. Middlehurst \& G. P. Kuiper), pp. 639-664. University of Chicago.

Yeomans, D. K. \& Chodas, P. W. 1994 Comet Shoemaker-Levy 9 in orbit about Jupiter. Bull. Amer. Astron. Soc. 26, 1566. 